\title{
THE UROKINASE RECEPTOR. PROTEIN STRUCTURE AND ROLE IN PLASMINOGEN ACTIVATOR AND CANCER INVASION
}

\author{
K. Danø, N. Behrendt, N. Brünner, V. Ellis, M. Ploug, C. Pyke \\ Einsen Laboratory, Rigshospitalet, Copenhagen, Denmark
}

Degradation of the extracellular matrix during cancer invasion is accomplished by a combination of proteolytic enzyme systems, including generation of plasmin catalyzed by the urokinase-type plasminogen activator (UPA). A specific receptor for UPA (UPAR) binds the enzyme to cell surfaces. UPAR is synthesized as a 313 amino acid residue polypeptide that is carboxyl-terminally processed and attached to the membrane by a glycosyl-phosphatidylinositol anchor. It consists of three homologous sequence repeats, the amino-terminal one being crucial for uPA-binding. The recent elucidation of the disulfide structure of this domain suggests that each of three UPAR domains are structurally related to snake venom $\alpha$-neurotoxins, such as $\alpha$ bungaratoxin, an assumption supported by several other findings. Isolated domain I has an at least 1500 -fold lower uPA-binding affinity than intact UPAR, possibly indicating that conformational changes induced by interdomain interactions within UPAR are important for the ligand binding. Concomitant binding of pro-uPA to UPAR and of plasminogen to unidentified binding sites at cell surfaces strongly enhances plasminogen activation, and surfaces of uPAR expressing cells thus appear to be preferential sites for plasminogen activation. The mechanism of this enhancement may be the formation of complexes in which there is a catalytically favorable assembly of pro-uPA/uPA and plasminogen/plasmin.

UPAR and UPA are consistently expressed at invasive foci in many types of human cancer. Often one or both of these molecules are expressed by non-malignant stromal cells such as macrophages or fibroblasts, indicating that these cells actively contribute to the invasive process. This active stromal cell participation in cancer invasion also includes expression of a variety of other components of proteolytic systems. It represents a new paradigm with important implications for basic cancer biology and cancer treatment. In addition the recent results in uPAR research have direct clinical implications. High levels of UPAR in breast and lung cancer tissue are, like high UPA and PAI-1 levels, associated with poor prognosis, and interference with the UPA:UPAR interaction represents a promising new approach for anti-invasive therapy. 